Research Article

\title{
Anisotropie der gesättigten Wasserleitfähigkeit in Böden der Hauptnaturräume Schleswig-Holsteins (Norddeutschland) unter Acker- und Grünlandnutzung
}

\section{Anisotropy of the hydraulic conductivity of soils in the geological regions of Schleswig-Holstein (Northern Germany) under arable und grassland use}

\author{
Anneka Mordhorst ${ }^{1 *}$, Heiner Fleige ${ }^{1}$, Iris Zimmermann ${ }^{1}$, Bernd Burbaum², Marek Filipinski ${ }^{2}$, \\ Eckhard Cordsen ${ }^{2}$, Rainer Horn ${ }^{1}$ \\ 1 Institut für Pflanzenernährung und Bodenkunde, Christian-Albrechts-Universität Kiel, Hermann Rodewaldstraße 2, 24118 Kiel, Deutschland \\ ${ }^{2}$ Landesamt für Landwirtschaft, Umwelt und ländliche Räume des Landes Schleswig-Holstein (LLUR), Hamburger Chaussee 25, 24220 \\ Flintbek, Deutschland \\ * Korrespondierende Autorin: amordhorst@soils.uni-kiel
}

Einreichung: 20. November 2018, überarbeitete Einreichung: 24. Jänner 2019, Annahme: 31. Jänner 2019

\section{Zusammenfassung}

Gegenwärtig diskutierte Phänomene, wie häufig auftretender Oberflächenabfluss, vermehrte Bodenerosion und verringerte Ertragssicherheit, lassen sich ansatzweise über Bodenstruktureigenschaften und eine daraus hervorgehende, räumlich unterschiedlich gesättigte Wasserleitfähigkeit des Bodens erklären. Hierzu wurden Datensätze aus 766 Profilen unter Acker- und Grünlandnutzung in den vier Hauptnaturräumen Schleswig Holsteins (Östliches Hügelland, Niedere und Hohe Geest sowie Marsch) zur gesättigten Wasserleitfähigkeit $\left(\mathrm{k}_{\mathrm{f}}\right)$ in vertikaler und horizontaler Richtung und damit deren Richtungsabhängigkeit (Anisotropie) bis in eine Tiefe von $60 \mathrm{~cm}$ untersucht. Die Ergebnisse zeigen eine verstärkte Anisotropie der $\mathrm{k}_{\mathrm{f}}$ in horizontaler Richtung an, die jedoch in Abhängigkeit vom geologischen Ausgangsgestein, Bodentyp und Tongehalt sowie von der Landnutzung variiert. Unter Ackernutzung weisen die Unterböden aller Hauptnaturräume im Mittel eine horizontale Anisotropie auf, die unterhalb der Pflugsohle ( $\leq 40 \mathrm{~cm}$ Tiefe) am stärksten ausgeprägt ist und auf Plattenstrukturbildungen als Folge der Bodenbewirtschaftung hindeuten. Unter Grünlandnutzung zeigen besonders die Oberböden eine horizontale Anisotropie, wobei diese in den beiden Geestregionen mit einem hohen Anteil an Viehwirtschaft auch im Unterboden sehr ausgeprägt ist. Anisotropieeffekte, insbesondere im Unterboden unter Ackernutzung, deuten sich auch bodentypspezifisch für Kolluvisole, Pseudogley-Parabraunerden und Pseudogleye an. Deren hohe Empfindlichkeit gegenüber anthropogenen Strukturänderungen (Plattenbildung) spiegelt sich im zeitlichen Verlauf der horizontalen $\mathrm{k}_{\mathrm{f}}$ wider, die über die letzten 30 Jahre im Östlichen Hügelland signifikant zugenommen hat.

Schlagworte: Landnutzung, Wasserleitfähigkeit, Plattengefüge, Unterbodenverdichtung, Anisotropie

\section{Summary}

Currently discussed phenomena like more frequent surface runoff after heavy rainfall events, increasing soil erosion and reduced yield stability can be explained to some extent by soil structure properties and a resulting, spatially differing saturated hydraulic conductivity of the soil. For this purpose, samples from 766 soil profiles under arable and grassland use in the four major natural environments of Schleswig-Holstein (Weichselian glacial area, Higher and Lower Geest, Marshland) were taken and, inter alia, the saturated hydraulic conductivity in vertical and horizontal direction and thereby its directional dependency (anisotropy) was evaluated down to the $60 \mathrm{~cm}$ depth. The results prove an enhanced anisotropy of the hydraulic conductivity in horizontal direction, with variations depending on the parent material, soil type and clay content, as well as the land use. The subsoils under arable land exhibit a horizontal anisotropy that is most distinct below the plow pan $(\leq 40 \mathrm{~cm}$ depth) and can be explained by the development of a platy soil structure as a consequence of the management (tillage). Soils under grassland use exhibit a horizontal anisotropy mainly in the topsoils, whereby it is additionally very pronounced in the subsoils of the two Geest regions with a high percentage of dairy farming. Such anisotropy effects, especially in the subsoils under arable land use, can be determined soil type specifically for the representative soils of the Weichselian glacial area: Anthrosols ("Colluvic"), Stagnic Luvisols and Stagnosols. The chronological sequence of the horizontal hydraulic conductivity mirrors their high sensitivity against anthropogenic soil structure changes (development of plates), which have clearly increased in the Weichselian glacial area during the latest 30 years.

Keywords: land use, hydraulic conductivity, platy structure, subsoil compaction, anisotropy 


\section{Einleitung}

Die Diskussion um die steigende Weltbevölkerung (9,7 Milliarden Menschen im Jahre 2050 nach Prognosen der UN 2017) bei ständig abnehmender landwirtschaftlicher Produktionsfläche (Abnahme ca. 70 ha d ${ }^{-1}$ in Deutschland (Statistisches Bundesamt, 2015) oder $300 \mathrm{~km}^{2} \mathrm{~d}^{-1}$ weltweit) verstärkt die Notwendigkeit, auch die Ursachen der Bodendegradation näher zu beleuchten, um hieraus neue Ansätze für eine nachhaltige Bodenbewirtschaftung abzuleiten. Durch ein nicht standortangepasstes Bodenmanagement, z. B. durch wiederholtes Befahren mit zu schweren landwirtschaftlichen Maschinen, wird der Degradation von Böden Vorschub geleistet, und es treten irreversible Veränderungen der Böden und deren Funktionen ein bzw. sind bereits eingetreten (Pagliai et al., 2004; Peth et al., 2006; Kim et al., 2010). Eine der wesentlichen Ursachen liegt in der immer noch ungebremsten Zunahme der Landmaschinengewichte auch weit über das z. B. auf Autobahnen zulässige Gewicht hinaus. Selbst bei Berücksichtigung der seitens der Industrie hervorgebrachten Argumente u. a. bezüglich einer effizienteren Bewirtschaftung, führen die mit dem Klimawandel zu erwartenden häufigeren Starkniederschläge (IPCC, 2014) zu weniger stabilen Böden, sodass die Gefahr von irreversiblen Bodenschäden erhöht sein wird (Jones et al., 2003; Horn und Fleige, 2009; Rogger et al., 2017).

Eine der negativen Folgen der Bodendegradation ist die Verstärkung des Oberflächenabflusses (z. B. Jones et al., 2003; Dörner und Horn, 2006; Bertolino et al., 2010), der mit als entscheidende Ursache für die in den letzten Jahren häufiger auftretenden Überflutungen an Flussläufen zu sehen ist. Nach van der Ploeg (1999) beruht die Zunahme des Oberflächenabflusses nicht nur auf der seit Mitte des letzten Jahrhunderts verstärkt durchgeführten Entwässerung landwirtschaftlich genutzter Flächen, sondern auch auf dem Verlust von Dauergrünland, das zunehmend als Ackerland umgebrochen wurde (19511989 von 15,7 auf $10,8 \%$ Westdeutschlands, van der Ploeg et al., 2002).

Hochwasserereignisse mit häufig katastrophalen Eigentumsschäden für die Bevölkerung sowie weitreichenden Folgen für die weitere Landnutzung kommen in den letzten Jahrzehnten sehr viel häufiger vor (Van der Ploeg und Schweigert, 2001; van der Ploeg et al., 2000 und 2002). Diese Überflutungen z. B. an Elbe, Oder und Donau, aber auch die als Folge von intensiveren Niederschlägen im Jahresverlauf entstehenden „Seenlandschaften“ lassen den Eindruck entstehen, dass die Böden tiefreichend vollständig wassergesättigt seien und dadurch diese Wassermassen nicht mehr aufgenommen und abgeführt werden können. Die Entnahme von Bodenproben nach solchen Extremereignissen zeigt, dass die Wassersättigung zwar im Oberboden tatsächlich gegeben ist, der Wassergehalt allerdings in den Bodentiefen $>40 \mathrm{~cm}$ wieder absinkt (Alaoui et al., 2018; Rogger et al., 2018), und folglich noch luftgefüllte Poren im Boden für eine zusätzliche Wasseraufnahme vorhanden wären. In verdichteten Böden ist die Wasserinfiltration jedoch am Übergang von der Ackerkrume zum Unterboden durch eine kompakte Pflugsohle aufgrund einer geringen vertikalen Wasserleitfähigkeit deutlich eingeschränkt (Bertolino et al., 2010; Dörner und Horn, 2006). Darüber hinaus können irreversible (plastische) Bodendeformationen eine entsprechende Umorientierung der richtungsabhängig leitenden Fließquerschnitte herbeiführen. Jede senkrechte Spannungsentlastung führt hierbei zu einer horizontalen Rissbildung (Horn, 1994), mit der Folge, dass nicht nur die vertikal durchgängigen Fließwege für Wasser oder Gas unterbrochen werden, sondern es gleichzeitig zur Bildung eines Plattengefüges kommt, das die Wasserinfiltration in tiefere Schichten reduziert. Zwischen den Plattenstrukturen steigt die horizontale Leitfähigkeit dagegen an, während durch den größeren Fließquerschnitt zwischen den Platten der kapillare Aufstieg infolge von wirksamen Kapillarsperren weitestgehend unterbunden sein kann (Beck-Broichsitter et al., 2017). Somit zeigen Plattenstrukturen unterhalb der Pflugsohle eine horizontale Anisotropie in der Fließbewegung auf (Dörner und Horn, 2009; Zink, 2009), die auch eine erhöhte Gefahr der Bodenerosion im geneigten Gelände nach sich zieht (Weißkopf et al., 2000; Bertolino et al., 2010; DeLaune und Sij, 2012; Horn, 2015; Bogunovic et al., 2018).

Inwieweit derartige negative Strukturänderungen in den landwirtschaftlich genutzten Böden in den vier Hauptnaturräumen Schleswig-Holsteins (Abbildung 1) nachweisbar sind, wurde anhand eines Datensatzes des LLUR S.-H. (Landesamt für Landwirtschaft, Umwelt und ländliche Räume des Landes SchleswigHolstein) zu vertikalen und horizontalen gesättigten Wasserleitfähigkeiten analysiert. Dabei wurden mögliche Einflussgrößen wie die Landnutzung, den Tongehalt und den Bodentyp auf die Wasserleitfähigkeit und deren Richtungsabhängigkeit berücksichtigt. 


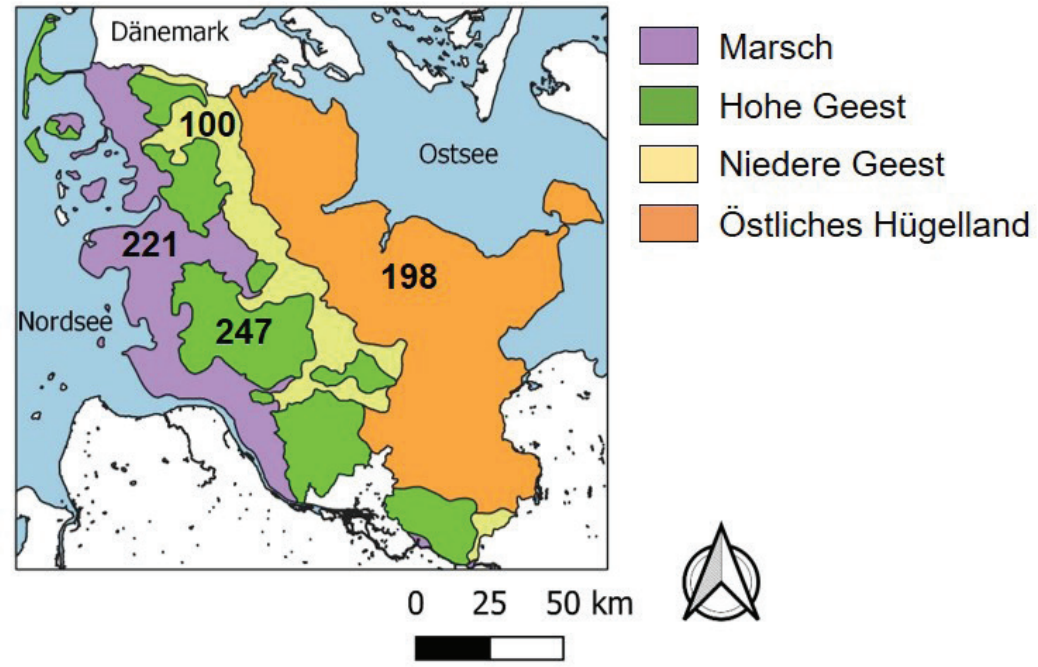

Abbildung 1. Übersichtskarte Schleswig-Holsteins (Norddeutschland) mit den vier Hauptnaturräumen und der jeweiligen Anzahl von analysierten Bodenprofilen in der Datenbank. Kartengrundlage: BKG (2010), verändert.

Figure 1. Geographical map of Schleswig-Holstein representing the 4 geological regions (North Germany) with the number of sampled soil profiles in the dataset. Map basis, modified: BKG (2010).

\section{Material und Methoden}

\subsection{Datengrundlage}

Im Rahmen unterschiedlicher Forschungsvorhaben des Landesamtes für Landwirtschaft, Umwelt und ländliche Räume des Landes SchleswigHolstein (LLUR S.-H.) und deren Vorgängerbehörden wurden in den vergangenen vier Jahrzehnten mehr als 900 Bodenprofile bis in mindestens $100 \mathrm{~cm}$ Bodentiefe bodenkundlich beschrieben und physikalische und chemische Bodenkenngrößen sowohl an ungestörten Proben, die entweder horizontal oder vertikal entnommen wurden, als auch an gestörten Proben bestimmt. Über die Datenbank lassen sich somit Aussagen über den bodentyp- sowie landnutzungsabhängigen Status quo der Böden in Bezug auf die bodenphysikalischen Eigenschaften ( $u$. a. gesättigte Wasserleitfähigkeit, $\mathrm{k}_{\mathrm{f}}$ ), Kohlenstoffvorräte (Mordhorst et al., 2018) sowie die Erosionsempfindlichkeit (Duttmann et al., 2016) treffen.

In der vorliegenden Arbeit wurden 766 Bodenprofile bis in $60 \mathrm{~cm}$ Tiefe im Hinblick auf die $\mathrm{k}_{\mathrm{f}}$-Werte des Bodens in horizontaler und vertikaler Richtung ausgewertet, um einen Überblick über die in den verschiedenen Hauptnaturräumen mit unterschiedlichem Ausgangsgestein und bei verschiedener Landbewirtschaftung (Acker- und Grünlandnutzung zum Zeitpunkt der Beprobung) auftretenden Spannbreiten der $\mathrm{k}_{\mathrm{f}}$-Werte, und vor allem der jeweils dominanten Fließrichtung des Wassers, im Boden zu erhalten
(Anisotropieeffekte). Die Daten wurden zu verschiedenen Zeitpunkten zwischen 1970-2015 für unterschiedliche Fragestellungen mit sechsfacher Wiederholung je Bodentiefe und -richtung erhoben und liegen als geometrische Mittelwerte in der Datenbank vor. Die Messungen der gesättigten Wasserleitfähigkeit erfolgten nach dem instationären Verfahren (Falling-head-Methode nach Hartge, 1966 bzw. Kretzschmar, 1996).

\subsection{Datenauswertung}

Die Auswertung der $\mathrm{k}_{\mathrm{f}}$-Werte in horizontaler und vertikaler Richtung in den Hauptnaturräumen Schleswig-Holsteins erfolgte nutzungs- (Grünland, Ackerland), bodentyp-, tiefen- $(\leq 40 \mathrm{~cm}, \leq 60 \mathrm{~cm})$ und tongehaltsspezifisch $(<12 \%$, $\geq 12 \%$ Ton), anhand geometrischer Mittelwerte (Anzahl der beprobten Horizonte (n): 3-73). Um den Einfluss der natürlichen Aggregatbildung auf die Wasserleitfähigkeit deutlicher zu dokumentieren, wurden die Datensätze je nach Tongehalt in die Klassen $<12 \%$ und größer $\geq 12 \%$ unterteilt. Diese Annahme beruht darauf, dass Aggregierungsprozesse in mitteleuropäischen Böden erst ab einem Tongehalt $\geq 12 \%$ an Bedeutung gewinnen (Horn, 1981). Das berechnete Verhältnis der Wasserleitfähigkeit in horizontaler und vertikaler Richtung beschreibt die bevorzugte Fließrichtung des Wassers im Boden. Während allgemein davon ausgegangen wird, dass isotrope Fließbedingungen in Böden (d. h. $\left.\mathrm{k}_{\mathrm{f}(\mathrm{h})} / \mathrm{k}_{\mathrm{f}(\mathrm{v})}=1\right)$ und damit 
gleichmäßige Wasserbewegungen in allen Raumrichtungen vorherrschen, definieren Quotienten $<1$ eine erhöhte Wasserleitfähigkeit in vertikaler Richtung (vertikal anisotrop), während Quotienten > 1 einen bevorzugt horizontalen Fluss (horizontal anisotrop) belegen. Die ermittelten Anisotropiewerte beschreiben folglich mittlere Bodenzustände, aus denen aktuelle hydraulische Funktionen abgeleitet werden können.

Die bodentypspezifische Auswertung erfolgte exemplarisch an ausgewählten repräsentativen Böden in den durch unterschiedliches Ausgangsmaterial geprägten Hauptnaturräumen Schleswig-Holsteins. Im Östlichen Hügelland (Gesamtfläche von $6740 \mathrm{~km}^{2}$, weichselkaltzeitlich geprägt) sind nach deutscher Bodensystematik (Ad-hoc-AG Boden, 2005) (Pseudogley-)Parabraunerden, Braunerden, Kolluvisole und Pseudogleye am häufigsten vertreten. Während in der Niederen Geest $\left(2010 \mathrm{~km}^{2}\right.$, Sanderebene) vor allem sandige Braunerden, (Gley-)Podsole und Gleye vorkommen, dominieren in der Hohen Geest $\left(3766 \mathrm{~km}^{2}\right.$, saalekaltzeitlich geprägt) lehmig-sandige und sandige, carbonatfreie Braunerden, (Gley-)Podsole und Pseudogleye. In der Marsch $\left(2854 \mathrm{~km}^{2}\right)$ gelten Kalk- und Klei- sowie Dwogund Knickmarschen als repräsentative Bodentypen. Eine ausführliche Auflistung der repräsentativen Bodentypen in den Hauptnaturräumen erfolgte in Mordhorst et al. (2018).

\subsection{Statistische Datenanalyse}

Die statistische Auswertung der Daten erfolgte mithilfe der Statistiksoftware R (R Core Team, 2018). Um eine Normalverteilung der $\mathrm{k}_{\mathrm{f}}$-Werte zu gewährleisten, wurden diese logarithmiert. Um den Einfluss der Entnahmerichtung (horizontal vs. vertikal), der Landnutzung (Ackerland vs. Grünland), der Tongehaltsklasse (<12\% vs. $\geq 12 \%$ Ton) und des Hauptnaturraumes (Östliches Hügelland, Niedere Geest, Hohe Geest und Marsch) auf die $\mathrm{k}_{\mathrm{f}}$-Werte zu untersuchen, gehen diese Varianten als Faktoren in die Varianzanalyse (ANOVA) ein. Signifikante Unterschiede der $\mathrm{k}_{\mathrm{f}}$-Werte zwischen den Varianten: Entnahmerichtung, Tongehaltsklasse und Nutzung wurden anschließend anhand des Tukey Post-hoc-Tests (zweiseitig) mit einem Signifikanzniveau von $\leq 0,05$ ermittelt.

\section{Ergebnisse}

Abbildung 2 informiert über die gesättigten Wasserleitfähigkeiten $\left(\mathrm{k}_{\mathrm{f}}\right)$ sowohl in horizontaler als auch vertikaler
Richtung bis in $40 \mathrm{~cm}$ Bodentiefe für Acker- und Grünlandstandorte aus den vier Hauptnaturräumen Schleswig-Holsteins. Ohne Berücksichtigung des geologischen Ausgangsmaterials weisen Böden unter ackerbaulicher Nutzung im Mittel signifikant höhere $\mathrm{k}_{\mathrm{f}}$-Werte in horizontaler als in vertikaler Richtung im Unterboden auf $(\mathrm{p}<0,01)$. Dabei reduziert sich die $\mathrm{k}_{\mathrm{f}}$ signifikant in beiden Richtungen mit höherem Tongehalt ( $\geq 12 \%$ Ton) auf Werte $<10 \mathrm{~cm} \mathrm{~d}^{-1}$ (Mittelwert) $(\mathrm{p}<0,001)$ und weist außerdem auf eine nach Ad-hoc-AG Boden (2005) „geringe“ Fließgeschwindigkeit hin. Die richtungsabhängige $\mathrm{k}_{\mathrm{f}}$ zeigt außerdem eine sich mit höherem Tongehalt stärker ausprägende Anisotropie unterhalb des gepflügten A-Horizontes. Unter Grünlandnutzung kommt es im Oberboden zu einer deutlichen horizontal ausgeprägten Anisotropie, die in Böden $<12 \%$ Ton signifikant $(\mathrm{p}<0,001)$ und in denen $\geq 12 \%$ Ton nur schwach ausgeprägt ist. Im Unterboden zeichnet sich dieser Effekt weniger deutlich ab bzw. es ist bei höherem Tongehalt sogar ein vertikal anisotropes Fließverhalten auszumachen $(\mathrm{p}<0,05)$.

Die geometrisch gemittelten horizontalen $\left(\mathrm{k}_{\mathrm{fhh}}\right)$ und vertikalen $\mathrm{k}_{\mathrm{f}(\mathrm{v})}$-Werte in den vier Hauptnaturräumen SchleswigHolsteins sind als Übersicht tabellarisch für Acker- (Tabelle 1) und Grünlandstandorte (Tabelle 2) aufgeführt. Wie zu erwarten, sind die durchlässigsten Böden in der Niederen Geest mit überwiegend sandigen Böden $(<12 \%$ Ton) zu finden, deren $\mathrm{k}_{\mathrm{f}}$-Werte im Mittel im ,sehr hohen “ Bereich (> $100 \mathrm{~cm} \mathrm{~d}^{-1}$, nach Ad-hoc-AG Boden, 2005) liegen. Unabhängig von der Landnutzung nehmen die mittleren $\mathrm{k}_{\mathrm{f}}$-Werte im Unterboden bis $\leq 60 \mathrm{~cm}$ Tiefe der tonarmen Standorte ( $<12 \%$ Ton) in der Reihenfolge Niedere Geest > Hohe Geest $\approx$ Östliches Hügelland (,mittel“ bis „hoch") > Marsch (,gering“) ab. Bei höherem Tongehalt ( $\geq 12 \%$ Ton) nähern sich die $\mathrm{k}_{\mathrm{f}}$-Werte im Unterboden weiter an, sind damit mit Ausnahme der Werte in der Niederen Geest signifikant geringer als bei $<12 \%$ Ton $(p<0,05)$ und liegen größtenteils unterhalb des „mittleren" Bereiches $\left(<40 \mathrm{~cm} \mathrm{~d}^{-1}\right)$.

In Hinblick auf die Richtungsabhängigkeit der $\mathrm{k}_{\mathrm{f}}$-Werte zeigt sich in Tabelle 1, dass im Mittel eine horizontale Anisotropie im Unterboden bis in $60 \mathrm{~cm}$ Tiefe der Ackerstandorte mit Tongehalten $<12 \%$ in allen Hauptnaturräumen vorliegt, die allerdings nicht signifikant ist. Steigt der Tongehalt ( $\geq 12 \%$ ), weisen ausschließlich die Unterböden aus der Hohen Geest eine signifikant ausgeprägte horizontale Anisotropie $\left(\mathrm{k}_{\mathrm{fh}} / \mathrm{k}_{\mathrm{f}(\mathrm{v})}>10\right)$ unter Ackernutzung bis in $60 \mathrm{~cm}$ Tiefe auf $(\mathrm{p}<0,05)$. Das horizontal anisotrope Fließverhalten verschärft sich für diese Böden mit höherem 


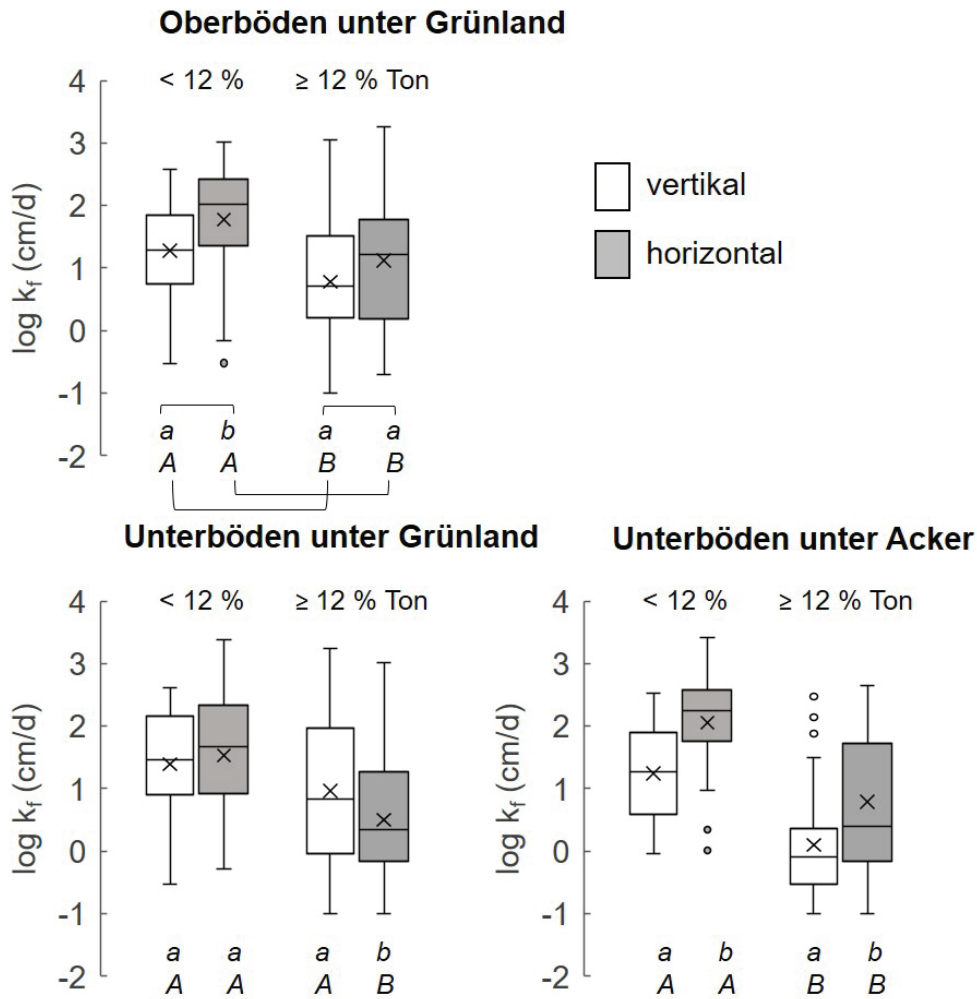

Abbildung 2. Vertikale und horizontale gesättigte hydraulische Leitfähigkeit $\left(\mathrm{k}_{\mathrm{f}}\right)$ in Oberböden unter Grünland (Ah-Horizonte) und in Unterböden $(\leq 40 \mathrm{~cm}$ Tiefe) unter Grünland- und Ackernutzung mit Tongehalten $<$ und $\geq 12 \%$. Die Abbildung beinhaltet alle untersuchten Bodenprofile in Schleswig-Holstein $(\mathrm{n}=507)$. In den Tabellen 1 und 2 sind die Anzahl der Wiederholungen pro Box-Plot dokumentiert (Datenbank: LLUR S.-H.). Signifikante Unterschiede zwischen horizontalen und vertikalen $\mathrm{k}_{\mathrm{f}}$-Werten sind mit unterschiedlichen Kleinbuchstaben (ab), diejenigen zwischen den Tongehaltsklassen mit unterschiedlichen Großbuchstaben (AB) bei einem Signifikanzniveau von 0,05 gekennzeichnet.

Figure 2. Vertical and horizontal hydraulic conductivity $\left(\mathrm{k}_{\mathrm{f}}\right)$ of top- (A-horizons for grassland) and subsoil horizons $(\leq 40 \mathrm{~cm}$ depth for arable and grassland soil horizons) with clay contents $<$ and $\geq 12 \%$ of all sampled soil profiles in Schleswig-Holstein (507 soil profiles in total). For number of replicates per boxplot see Tables 1 and 2 (Data basis: LLUR S.-H.). Significant differences between $\mathrm{k}_{\mathrm{f}}$ values are documented by lowercases (ab), those between clay content classes by uppercases $(\mathrm{AB})$ with a significance level of 0.05 .

Tongehalt und ist für Unterbodenhorizonte unterhalb der Pflugsohle, d. h. $\leq 40 \mathrm{~cm}$ Tiefe, betragsmäßig, jedoch nicht signifikant, höher $\left(\mathrm{k}_{\mathrm{f}(\mathrm{h})} / \mathrm{k}_{\mathrm{f}(\mathrm{v})}>25\right)$ als in $\leq 60 \mathrm{~cm}$ Tiefe. Unter Grünlandnutzung liegen in den besonders intensiv genutzten Böden der Niederen und Hohen Geest die Anisotropie-Werte sowohl im Oberboden (A-Horizonte) als auch im Unterboden $\leq 60 \mathrm{~cm}$ deutlich $>1$ (Tabelle 2). Für die Oberböden der Hohen Geest sind Unterschiede in der horizontalen und vertikalen $\mathrm{k}_{\mathrm{f}}$ dabei signifikant $(<12 \%$ Ton: $\mathrm{p}<0,001,>12 \%$ Ton: $\mathrm{p}<0,1)$. Insgesamt ist die horizontale Anisotropie in Böden $\geq 12 \%$ größer als in denen mit $<12 \%$ Ton, wohingegen die vergleichsweise tonhaltigeren Unterböden des Östlichen Hügellands (nicht signifikant) und der Marsch $(\mathrm{p}<0,05)$ ein eher vertikal anisotropes Fließverhalten bis $\leq 60 \mathrm{~cm}$ Tiefe aufweisen. In der Niederen Geest sind die tonangereicherten Böden $\geq 12 \%$
Ton jedoch wegen ihrer geringen flächenmäßigen Ausbreitung und der damit zusammenhängenden geringen Beprobungshäufigkeit $(\mathrm{n}<6)$ als nicht-repräsentativ anzusehen. Differenziert man weiterhin nach den besonders empfindlichen Bodentypen und Bodenhorizonten, dann können vor allem die nicht pedogen/geogen verdichteten $\mathrm{Sw}-\mathrm{Al}$ Horizonte der Pseudogley-Parabraunerden, die M-Horizonte der Kolluvisole sowie tendenziell die Sw-Horizonte der primären und sekundären Pseudogleye unter Ackernutzung, aber z. T. auch unter Grünlandnutzung im Mittel mit einem bevorzugten Wasserfluss in horizontaler Richtung definiert werden (Abbildung 3). Signifikante Unterschiede treten dabei nur für den Bodentyp „Kleimarsch“ hinsichtlich der Landnutzung ( $\mathrm{p}<0,001)$ und Entnahmerichtung $(\mathrm{p}<0,01)$ auf, die wie auch bei der Kalkmarsch eine vertikale Anisotropie aufzeigt. 
Tabelle 1. Horizontale und vertikale gesättigte Wasserleitfähigkeit $\left(\mathrm{k}_{\mathrm{f}}\right)$ von Unterböden unter Ackernutzung $(\leq 40 \mathrm{~cm} ; \leq 60 \mathrm{~cm}$ Tiefe $)$ mit Tongehalten $<$ und $\geq 12 \%$ in den vier Hauptnaturräumen von Schleswig-Holstein. $\mathrm{n}=$ Anzahl der Bodenproben, Anisotropie = Verhältnis aus horizontaler zu vertikaler Wasserleitfähigkeit (geometrische Mittelwerte), MW Ton = arithmetischer Mittelwert des Tongehalts, GeoM = Geometrisches Mittel, $\mathrm{n}<3$ (-) (Datenbank: LLUR S.-H). Signifikante Unterschiede zwischen horizontalen und vertikalen $\mathrm{k}_{\mathrm{f}}$-Werten sind mit unterschiedlichen Kleinbuchstaben (ab), diejenigen zwischen den Tongehaltsklassen mit unterschiedlichen Großbuchstaben (AB) bei einem Signifikanzniveau von 0,05 gekennzeichnet. Table 1 . Horizontal and vertical hydraulic conductivity $\left(\mathrm{k}_{\mathrm{f}}\right)$ of arable subsoils $(\leq 40 \mathrm{~cm} ; \leq 60 \mathrm{~cm}$ depth) with clay contents $<12 \%$ and $\geq 12 \%$ in the four geological regions of Schleswig-Holstein. $\mathrm{n}=$ number of samples, Anisotropy = ratio between horizontal to vertical $\mathrm{k}_{\mathrm{f}}$ values $($ geometric mean), MW Ton = arithmetic mean of clay content, GeoM = geometric mean, $\mathrm{n}<3$ (-) (data basis: LLUR S.-H.). Significant differences between $\mathrm{k}_{\mathrm{f}}$ values are documented by lowercases $(\mathrm{ab})$, those between clay content classes by uppercases $(\mathrm{AB})$ with a significance level of 0.05 .

\begin{tabular}{|c|c|c|c|c|c|c|c|c|c|c|c|c|c|c|c|}
\hline \multirow{4}{*}{ Tiefe } & \multirow{4}{*}{ Hauptnaturraum } & \multicolumn{7}{|c|}{$<12 \%$ Ton } & \multicolumn{7}{|c|}{$\geq 12 \%$ Ton } \\
\hline & & \multicolumn{3}{|c|}{ horizontal (h) } & \multicolumn{3}{|c|}{ vertikal (v) } & \multirow{2}{*}{$\begin{array}{c}\mathrm{h} / \mathrm{v} \\
\begin{array}{l}\text { Aniso- } \\
\text { tropie }\end{array}\end{array}$} & \multicolumn{3}{|c|}{ horizontal (h) } & \multicolumn{3}{|c|}{ Vertikal (v) } & \multirow{2}{*}{$\begin{array}{c}\mathrm{h} / \mathrm{v} \\
\begin{array}{c}\text { Aniso } \\
\text { tropic }\end{array}\end{array}$} \\
\hline & & $\begin{array}{c}\text { MW } \\
\text { Ton }\end{array}$ & $\begin{array}{c}\text { GeoM } \\
\mathbf{k}_{\mathrm{f}}\end{array}$ & $n$ & $\begin{array}{c}\text { MW } \\
\text { Ton }\end{array}$ & $\begin{array}{c}\text { GeoM } \\
\mathbf{k}_{\mathrm{f}}\end{array}$ & $n$ & & $\begin{array}{l}\text { MW } \\
\text { Ton }\end{array}$ & $\begin{array}{c}\text { GeoM } \\
\mathbf{k}_{\mathrm{f}}\end{array}$ & $n$ & $\begin{array}{l}\text { MW } \\
\text { Ton }\end{array}$ & $\begin{array}{c}\text { GeoM } \\
\mathbf{k}_{\mathrm{f}}\end{array}$ & $n$ & \\
\hline & & $(\%)$ & $\left(\mathrm{cm} \mathrm{d}^{-1}\right)$ & $(-)$ & $(\%)$ & $\left(\mathrm{cm} \mathrm{d}^{-1}\right)$ & $(-)$ & $(-)$ & $(\%)$ & $\left(\mathrm{cm} \mathrm{d}^{-1}\right)$ & $(-)$ & $(\%)$ & $\left(\mathrm{cm} \mathrm{d}^{-1}\right)$ & $(-)$ & $(-)$ \\
\hline \multirow{5}{*}{$\begin{array}{l}\text { Unterboden- } \\
\text { horizonte } \\
(\leq 40 \mathrm{~cm})\end{array}$} & Östliches Hügelland & 6,3 & $105,7 \mathrm{aA}$ & 16 & 5,4 & $30,8 \mathrm{aA}$ & 6 & 3,4 & 21,5 & $7,8 \mathrm{aA}$ & 29 & 24,3 & $1,9 \mathrm{aA}$ & 12 & 4,0 \\
\hline & Niedere Geest & - & - & - & - & - & - & - & - & - & - & - & - & - & - \\
\hline & Hohe Geest & 5,3 & $177,7 \mathrm{aA}$ & 10 & 5,3 & $28,2 \mathrm{aA}$ & 11 & 6,3 & 20,2 & $14,2 \mathrm{aA}$ & 3 & 17,0 & $0,6 \mathrm{aB}$ & 5 & 25,4 \\
\hline & Marsch & - & - & - & 8,2 & $3,6 \mathrm{~A}$ & 6 & - & 23,1 & $0,9 \mathrm{a}$ & 6 & 28,5 & $1,0 \mathrm{aA}$ & 32 & 0,9 \\
\hline & Gesamt & 6,1 & 112,8 & 29 & 6,0 & 17,3 & 27 & 6,5 & 21,5 & 6,1 & 39 & 26,1 & 1,2 & 50 & 4,9 \\
\hline \multirow{5}{*}{$\begin{array}{l}\text { Unterboden- } \\
\text { horizonte } \\
(\leq 60 \mathrm{~cm})\end{array}$} & Östliches Hügelland & 6,1 & $89,7 \mathrm{aA}$ & 43 & 5,4 & $53,5 \mathrm{aA}$ & 16 & 1,7 & 22,1 & $6,2 \mathrm{aB}$ & 89 & 23,8 & $7,1 \mathrm{aB}$ & 38 & 0,9 \\
\hline & Niedere Geest & 3,6 & 259,8 a & 8 & 3,8 & $127,3 \mathrm{aA}$ & 10 & 2,0 & - & - & - & 14,1 & $14,1 \mathrm{aA}$ & 3 & - \\
\hline & Hohe Geest & 5,6 & $119,0 \mathrm{aA}$ & 30 & 5,7 & $43,6 \mathrm{aA}$ & 48 & 2,7 & 17,8 & $12,4 \mathrm{aB}$ & 17 & 18,6 & $1,2 \mathrm{bB}$ & 22 & 10,2 \\
\hline & Marsch & 9,2 & $9,0 \mathrm{aA}$ & 7 & 8,5 & $7,2 \mathrm{aA}$ & 17 & 1,3 & 23,6 & $0,7 \mathrm{aA}$ & 13 & 29,1 & $1,1 \mathrm{aB}$ & 73 & 0,7 \\
\hline & Gesamt & 5,9 & 90,6 & 88 & 6,0 & 36,0 & 91 & 2,5 & 21,6 & 5,6 & 121 & 25,6 & 2,0 & 136 & 2,8 \\
\hline
\end{tabular}

Abbildung 4 zeigt die zeitliche Änderung der $\mathrm{k}_{\mathrm{f}}$ in Unterböden des Östlichen Hügellands. Für die anderen Hauptnaturräume fehlen geeignete Datensätze, um eine kontinuierliche Entwicklung der $\mathrm{k}_{\mathrm{f}}$-Werte über den Beprobungszeitraum von 1970-2015 zu dokumentieren. Insbesondere die Unterböden $\leq 40 \mathrm{~cm}$, aber auch diejenigen $\leq 60 \mathrm{~cm}$ weisen bei Tongehalten $>12 \%$ eine mit der Zeit signifikant $\left(\mathrm{R}^{2}=0,73\right.$ bzw. $\left.0,74, \mathrm{p}<0,001\right)$ größer werdende horizontale Wasserleitfähigkeit auf. Bei Tongehalten $\leq 12 \%$ lässt sich dieser positive Trend weniger deutlich nachweisen $\left(R^{2}=0,24\right.$ bzw. 0,25).

Um einen Hinweis auf die besonders empfindlichen Böden bzw. Bodenhorizonte zu erhalten, ist in Abbildung 5 die zeitliche Veränderung der $k_{\mathrm{f}}$ in horizontaler Richtung für Pseudogley-Parabraunerden und Norm-Pseudogleye in den beiden untersuchten Bodentiefen über die Zeit dargestellt. Der zeitliche Trend weist dabei auf signifikante $\mathrm{Zu}$ - nahmen der horizontalen $\mathrm{k}_{\mathrm{f}}$ in $(\mathrm{Sw})$-Al- $(\mathrm{p}<0,01)$ sowie Sw-Horizonten $(\mathrm{p}<0,001)$ bis in die Bodentiefe $\leq 60 \mathrm{~cm}$ mit der Zeit hin.

\section{Diskussion}

Bodenfunktionen wie die hydraulische Leitfähigkeit, aber auch die Luftpermeabilität und Gasdiffusion sowie die Wärmeleitfähigkeit sind als richtungsabhängige Werte zu betrachten. Generell sind in Böden keine isotropen Fließund Diffusionsbedingungen zu erwarten, da diese nur unter der Annahme eines Kugelmodells von gleich großen Kugeln mit 12 Kontaktpunkten (hexagonal dichteste Packung, Hartge und Horn, 2014) vorherrschen. In allen anderen Konfigurationen kommt es zu einer mehr oder weniger stark ausgeprägten Anisotropie. Solche Abwei- 

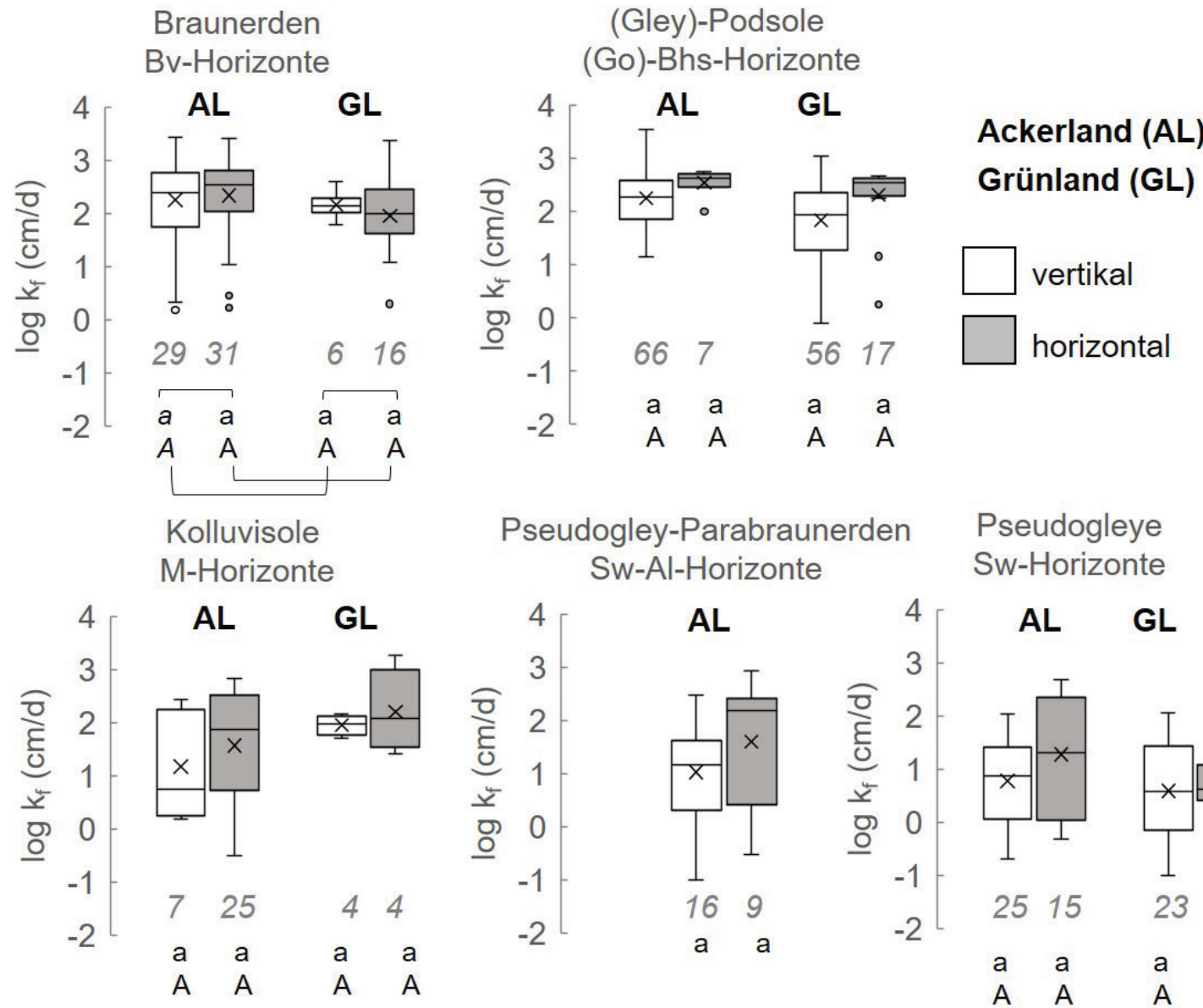

\section{Pseudogley-Parabraunerden Sw-Al-Horizonte}

Pseudogleye
Sw-Horizonte
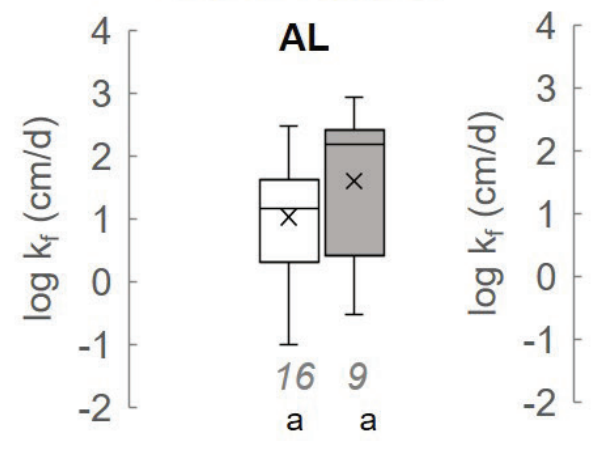

AL GL

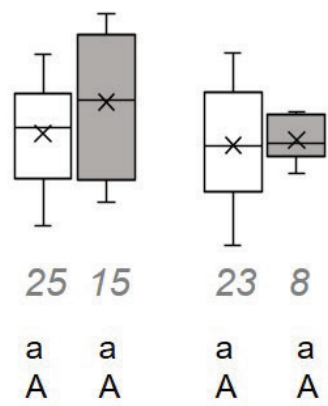

Kleimarschen Go-Horizonte

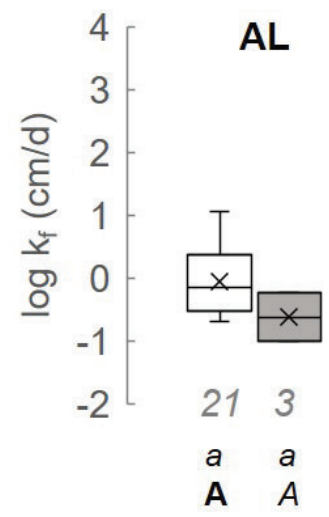

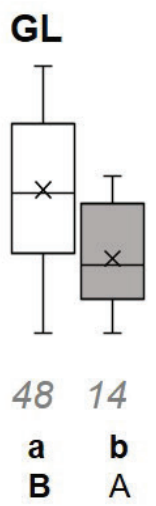

Kalkmarschen eGo-Horizonte

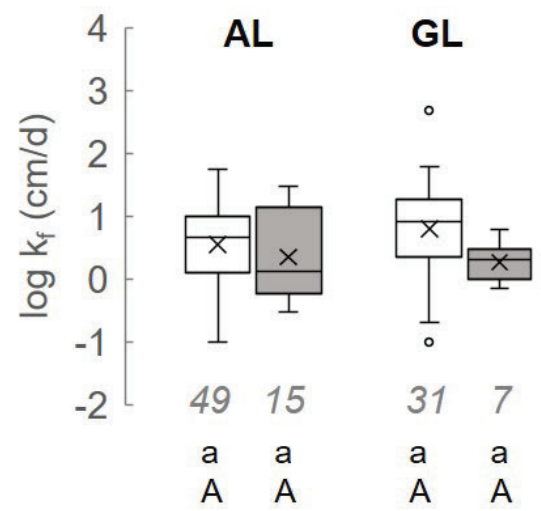

Abbildung 3. Vertikale und horizontale hydraulische gesättigte Wasserleitfähigkeit $\left(\mathrm{k}_{\mathrm{f}}\right)$ der Unterbodenhorizonte $(\leq 60 \mathrm{~cm}$ Tiefe) von repräsentativen Bodentypen Schleswig-Holsteins unter Acker- (A) und Grünlandnutzung (G). Zahlen unter den Boxplots geben die Anzahl der Proben wieder (Datenbank: LLUR S.-H.). Signifikante Unterschiede zwischen horizontalen und vertikalen $\mathrm{k}_{\mathrm{f}}$-Werten sind mit unterschiedlichen Kleinbuchstaben (ab), diejenigen zwischen den Landnutzungstypen mit unterschiedlichen Großbuchstaben (AB) bei einem Signifikanzniveau von 0,05 gekennzeichnet.

Figure 3. Vertical and horizontal hydraulic conductivity $\left(\mathrm{k}_{\mathrm{f}}\right)$ of subsoil horizons ( $\leq 60 \mathrm{~cm}$ depth) from representative soil types in Schleswig-Holstein under arable land (A) and grassland use $(\mathrm{G})$. Number of samples is given below the boxplots (Data basis: LLUR S.-H.) Significant differences between $k_{f}$ values are documented by lowercases $(\mathrm{ab})$, those between land use types by uppercases $(\mathrm{AB})$ with a significance level of 0.05 . 
Tabelle 2. Horizontale und vertikale gesättigte Wasserleitfähigkeit $\left(\mathrm{k}_{\mathrm{f}}\right)$ von Unterböden unter Grünlandnutzung $(\leq 40 \mathrm{~cm} ; \leq 60 \mathrm{~cm}$ Tiefe $)$ mit Tongehalten $<$ und $\geq 12 \%$ in den vier Hauptnaturräumen von Schleswig-Holstein. $\mathrm{n}=$ Anzahl der Bodenproben, Anisotropie = Verhältnis aus horizontaler zu vertikaler Wasserleitfähigkeit (geometrische Mittelwerte), MW Ton = arithmetischer Mittelwert, GeoM = Geometrisches Mittel, $\mathrm{n}<3$ (-), Datenbank: LLUR S.-H. Signifikante Unterschiede zwischen horizontalen und vertikalen $\mathrm{k}_{\mathrm{f}}-$ Werten sind mit unterschiedlichen Kleinbuchstaben (ab), diejenigen zwischen den Tongehaltsklassen mit unterschiedlichen Großbuchstaben (AB) bei einem Signifikanzniveau von 0,05 bzw. 0,1 (Buchstaben in Klammern gesetzt) gekennzeichnet.

Table 2 . Horizontal and vertical hydraulic conductivity $\left(\mathrm{k}_{\mathrm{f}}\right)$ of grassland top- and subsoils $(\leq 40 \mathrm{~cm} ; \leq 60 \mathrm{~cm}$ depth) with clay contents $<12 \%$ and $\geq 12 \%$ of the four geological regions of Schleswig-Holstein. $\mathrm{n}=$ number of samples, Anisotropy = ratio between horizontal to vertical ks values (geometric mean), MW Ton = arithmetic mean, GeoM = geometric mean, $\mathrm{n}<3(-)$, data basis: LLUR S.-H. Significant differences between $\mathrm{k}_{\mathrm{f}}$ values are documented by lowercases $(\mathrm{ab})$, those between clay content classes by uppercases $(\mathrm{AB})$ with a significance level of 0.05 or 0.1 (letters in brackets).

\begin{tabular}{|c|c|c|c|c|c|c|c|c|c|c|c|c|c|c|c|}
\hline \multirow{4}{*}{ Tiefe } & \multirow{4}{*}{ Hauptnaturraum } & \multicolumn{7}{|c|}{$<12 \%$ Ton } & \multicolumn{7}{|c|}{$\geq 12 \%$ Ton } \\
\hline & & \multicolumn{3}{|c|}{ horizontal (h) } & \multicolumn{3}{|c|}{ vertikal (v) } & \multirow{2}{*}{$\begin{array}{c}\mathrm{h} / \mathrm{v} \\
\begin{array}{c}\text { Aniso- } \\
\text { tropie }\end{array}\end{array}$} & \multicolumn{3}{|c|}{ horizontal (h) } & \multicolumn{3}{|c|}{ vertikal (v) } & \multirow{2}{*}{$\begin{array}{c}\mathrm{h} / \mathrm{v} \\
\begin{array}{c}\text { Aniso } \\
\text { tropi }\end{array}\end{array}$} \\
\hline & & $\begin{array}{l}\text { MW } \\
\text { Ton }\end{array}$ & $\begin{array}{c}\text { GeoM } \\
\mathbf{k}_{\mathrm{f}}\end{array}$ & $n$ & $\begin{array}{l}\text { MW } \\
\text { Ton }\end{array}$ & $\begin{array}{c}\text { GeoM } \\
\mathbf{k}_{\mathrm{f}}\end{array}$ & $n$ & & $\begin{array}{l}\text { MW } \\
\text { Ton }\end{array}$ & $\begin{array}{c}\text { GeoM } \\
\mathbf{k}_{\mathrm{f}}\end{array}$ & $n$ & $\begin{array}{l}\text { MW } \\
\text { Ton }\end{array}$ & GeoM k $k_{f}$ & $n$ & \\
\hline & & $(\%)$ & $\left(\mathrm{cm} \mathrm{d}^{-1}\right)$ & $(-)$ & $(\%)$ & $\left(\mathrm{cm} \mathrm{d}^{-1}\right)$ & $(-)$ & $(-)$ & $(\%)$ & $\left(\mathrm{cm} \mathrm{d}^{-1}\right)$ & $(-)$ & $(\%)$ & $\left(\mathrm{cm} \mathrm{d}^{-1}\right)$ & $(-)$ & $(-)$ \\
\hline \multirow{5}{*}{$\begin{array}{c}\text { Ah- } \\
\text { Horizonte } \\
(\leq 40 \mathrm{~cm})\end{array}$} & Östliches Hügelland & 6,0 & $50,5 \mathrm{aA}$ & 28 & 6,9 & $85,4 \mathrm{aA}$ & 15 & 0,6 & 23,2 & $35,1 \mathrm{aA}$ & 23 & 22,1 & $30,8 \mathrm{aA}$ & 12 & 1,2 \\
\hline & Niedere Geest & 5,8 & $125,4 \mathrm{a}$ & 12 & 4,5 & $36,4 \mathrm{a}$ & 24 & 3.4 & - & - & - & - & - & - & - \\
\hline & Hohe Geest & 5,8 & 84,5 aA & 36 & 6,8 & $12,6 \mathrm{bA}$ & 71 & 6,7 & 17,3 & 62,7 (a)A & 4 & 15,1 & 2,0 (b)A & 8 & 31,9 \\
\hline & Marsch & 7,5 & $6,6 \mathrm{aA}$ & 8 & 8,1 & $4,3 \mathrm{aA}$ & 7 & 1,5 & 28,1 & $1,9 \mathrm{aA}$ & 15 & 29,8 & $4,2 \mathrm{aA}$ & 45 & 0,5 \\
\hline & Gesamt & 6,0 & 59,1 & 84 & 6,4 & 18,8 & 117 & 3,1 & 24,4 & 13,1 & 42 & 26,1 & 6,0 & 67 & 2,2 \\
\hline \multirow{5}{*}{$\begin{array}{l}\text { Unter- } \\
\text { boden- } \\
\text { horizonte } \\
(\leq 40 \mathrm{~cm})\end{array}$} & Östliches Hügelland & 5,5 & $61,5 \mathrm{aA}$ & 14 & 6,3 & $151,6 \mathrm{aA}$ & 5 & 0,4 & 22,0 & $14,2 \mathrm{aB}$ & 10 & 28,2 & $19,6 \mathrm{aA}$ & 10 & 0,7 \\
\hline & Niedere Geest & - & - & - & - & - & - & - & - & - & - & - & - & - & - \\
\hline & Hohe Geest & 5,1 & $29,2 \mathrm{aA}$ & 6 & 5,0 & 28,7 aA & 14 & 1,0 & 21,5 & $3,8 \mathrm{aA}$ & 4 & 18,1 & $0,9 \mathrm{aB}$ & 11 & 4,1 \\
\hline & Marsch & 9,2 & $4,7 \mathrm{aA}$ & 8 & 8,2 & $9,2 \mathrm{aA}$ & 14 & 0,5 & 32,4 & $2,0 \mathrm{aA}$ & 46 & 32,8 & $11,6 \mathrm{bA}$ & 107 & 0,2 \\
\hline & Gesamt & 6,3 & 33,6 & 32 & 6,5 & 24,8 & 34 & 1,4 & 29,6 & 3,2 & 61 & 30,9 & 9,1 & 132 & 0,3 \\
\hline \multirow{5}{*}{$\begin{array}{l}\text { Unter- } \\
\text { boden- } \\
\text { horizonte } \\
(\leq 60 \mathrm{~cm})\end{array}$} & Östliches Hügelland & 5,0 & $62,3 \mathrm{aA}$ & 22 & 6,6 & $105,8 \mathrm{aA}$ & 9 & 0,6 & 22,9 & $16,9 \mathrm{aA}$ & 27 & 27,4 & $18,9 \mathrm{aA}$ & 27 & 0,9 \\
\hline & Niedere Geest & 3,7 & $402,6 \mathrm{aA}$ & 10 & 3,8 & $147,2 \mathrm{aA}$ & 12 & 2,7 & 15,6 & 822,9 aA & 3 & 20,6 & $1,5 \mathrm{aB}$ & 6 & 552,0 \\
\hline & Hohe Geest & 4,7 & 61,8 aA & 15 & 5,5 & $18,1 \mathrm{aA}$ & 49 & 3,4 & 22,1 & $4,1 \mathrm{aA}$ & 8 & 19,1 & $1,1 \mathrm{aB}$ & 38 & 3,7 \\
\hline & Marsch & 8,2 & $3,6 \mathrm{aA}$ & 12 & 7,7 & $8,7 \mathrm{aA}$ & 30 & 0,4 & 33,2 & $1,8 \mathrm{aA}$ & 76 & 32,8 & 8,6 bA & 186 & 0,2 \\
\hline & Gesamt & 5,3 & 47,7 & 59 & 6,0 & 21,9 & 100 & 2,2 & 29,5 & 3,9 & 114 & 29,9 & 6,6 & 257 & 0,6 \\
\hline
\end{tabular}

chungen von der Partikelordnung werden zudem durch natürliche (u. a. geogene oder pedogene Verdichtungen, Gefüge-/Strukturbildung, Bioturbation) als auch anthropogene Vorgänge (mechanische Verdichtung durch externe Auflasten) verursacht. So besitzen Bodenhorizonte mit prismatischer Struktur eine vorrangig vertikale Anisotropie, hervorgerufen durch die Entwicklung von sekundären groben Poren zwischen den Aggregaten (Risse). Der Tongehalt, ab dem mit einer deutlichen Strukturbildung gerech- net werden kann, wird häufig bei $12 \%$ für mitteleuropäische Bodenverhältnisse festgelegt, da bei höheren Gehalten ausgeprägte Quell-/Schrumpfungsprozesse mit einer deutlichen Zunahme der Leitfunktionen sowie der Bodenstabilität einhergehen (Horn, 1981 und 1994). Dies spiegelt sich insbesondere für die untersuchten Unterböden aus der Marsch mit hohem Quellungs- und Schrumpfungspotenzial wider, die unabhängig von der Landnutzung eine vertikal ausgerichtete Anisotropie der hydraulischen 
Unterbodenhorizonte $\leq \mathbf{4 0} \mathrm{cm}$ Tiefe
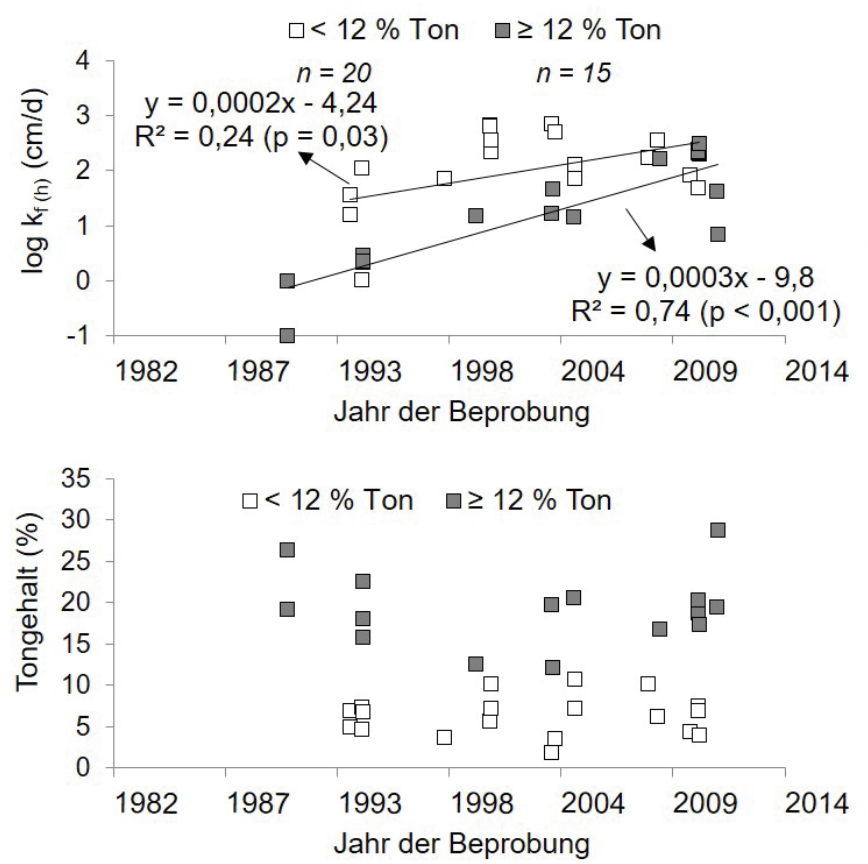

Unterbodenhorizonte $\leq 60 \mathrm{~cm}$ Tiefe
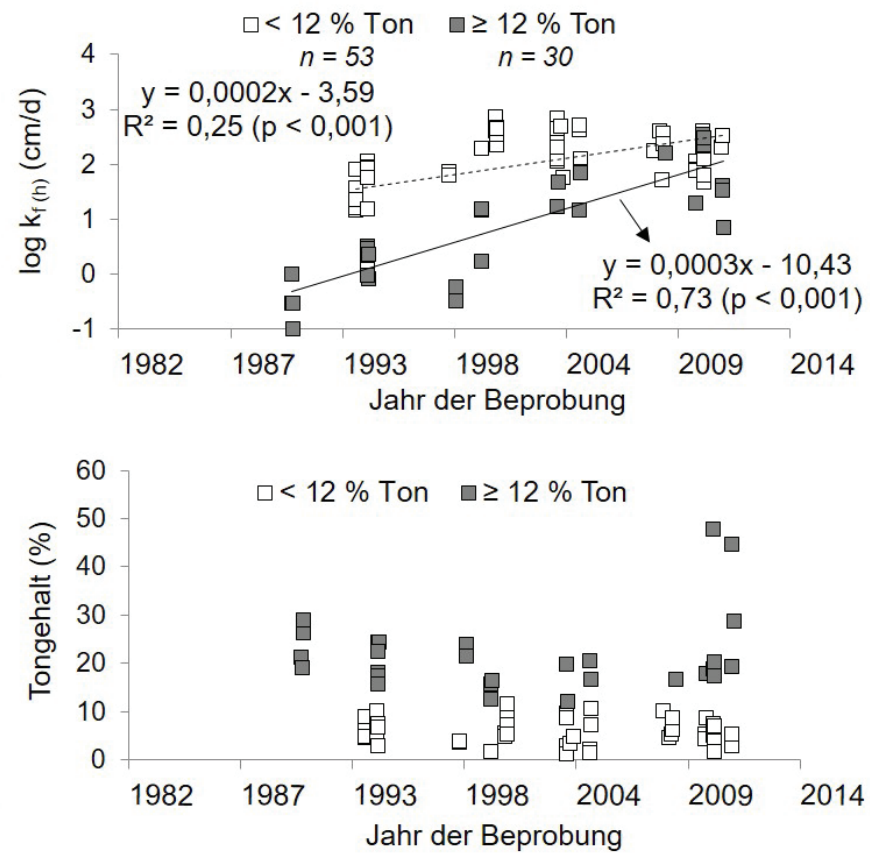

Abbildung 4. Horizontale hydraulische Leitfähigkeiten $\left(\mathrm{k}_{\mathrm{f}(\mathrm{h})}\right)$ und Tongehalte von nicht von Natur aus verdichteten Unterbodenhorizonten der untersuchten Böden aus dem Östlichen Hügelland Schleswig-Holsteins unter Ackernutzung in $\leq 40 \mathrm{~cm}$ und $60 \mathrm{~cm}$ Tiefe im Zeitverlauf (Datenbank: LLUR S.-H.).

Figure 4. Horizontal hydraulic conductivity $\left(\mathrm{k}_{\mathrm{f}(\mathrm{h})}\right)$ and clay contents of arable, not naturally compacted subsoil horizons of the analyzed soils of the Weichselian glacial area of Schleswig-Holstein in $\leq 40 \mathrm{~cm}$ and $\leq 60 \mathrm{~cm}$ over time (data basis: LLUR S.-H.).

Leitfähigkeit für Böden $\geq 12 \%$ Ton aufweisen (Tabellen 1 und 2, Abbildung 4). Die Ergebnisse legen außerdem nahe, dass sich eine stärker ausgeprägte vertikale Fließrichtung durch strukturbedingte Sekundärporen nur in tonreicheren Unterböden ( $\geq 12 \%$ Ton) unter Grünlandnutzung ausgebildet hat, die im Vergleich zu denen unter Ackernutzung mutmaßlich geringeren mechanischen Belastungen ausgesetzt sind bzw. deren externen Spannungseinträge weniger tief reichen, sodass Strukturbildungsprozesse im Unterboden ungestörter ablaufen konnten.

Ursachen einer verstärkt horizontal ausgeprägten Anisotropie können hingegen sowohl natürlich als auch anthropogener Herkunft sein. Eine Differenzierung ist über die Betrachtung einzelner Bodenhorizonte und Tongehaltsklassen annäherungsweise möglich. Hier kann beispielsweise die festgestellte schwach ausgeprägte horizontale Anisotropie in Bhs-Horizonten sandiger (Gley-)Podsole mitunter auch auf eine pedogene Verfestigung (Kittgefüge) zurückgeführt werden, während nicht natürlich verdichtete Horizonte wie (Sw-)Al- der (Pseudogley-)Parabrauner- den, Sw- oder M-Horizonte der Pseudogleye bzw. der Kolluvisole auf anthropogen bedingte Strukturänderungen hindeuten (Abbildung 4).

Werden die Ergebnisse der Wasserleitfähigkeiten in horizontaler und vertikaler Richtung in den Unterböden unter Ackernutzung betrachtet, so kann insbesondere die innerhalb von $40 \mathrm{~cm}$ Bodentiefe ausgeprägte horizontale Anisotropie als Indiz dafür gelten, dass es unterhalb des gepflügten A-Horizontes mit seinen sehr stark wechselnden, instabilen Strukturverhältnissen infolge der über Jahrzehnte durchgeführten Pflugarbeit und wiederholten Befahrungen (einschließlich der durch Schlupf hervorgerufenen Scherung) zu einer verstärkten Einregelung und vermehrt auftretenden Plattenstrukturbildung gekommen ist (Horn und Peth, 2011; Glinski et al., 2011; Holthusen et al., 2018). Bezüglich der Marschböden, die wie bereits erwähnt keine horizontale Anisotropie im Unterboden aufweisen, ist zusätzlich zu berücksichtigen, dass die Beprobungen Mitte der 1990er-Jahre weitgehend abgeschlossen waren, sodass anthropogen bedingte Strukturänderungen 
(Sw)-Al-Horizonte

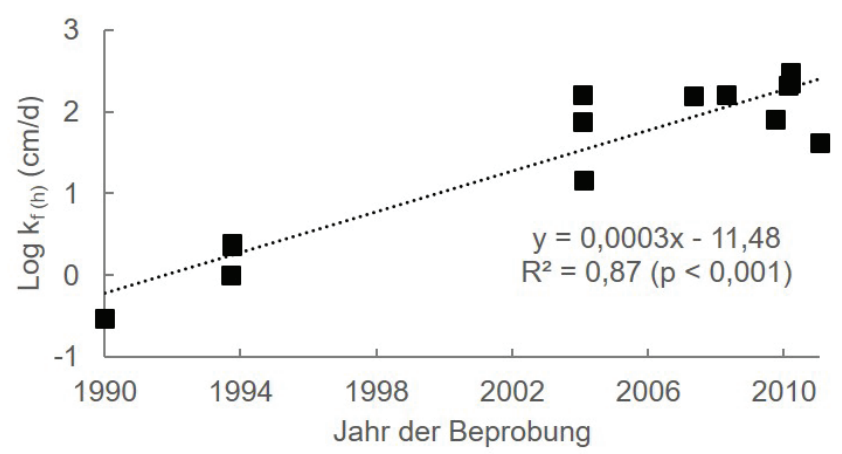

Sw-Horizonte

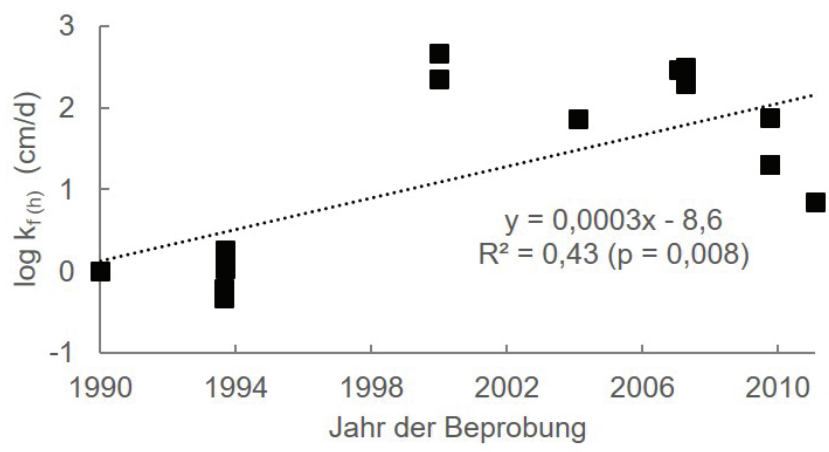

Abbildung 5. Änderung der horizontalen hydraulischen Leitfähigkeit $\left(\mathrm{k}_{\mathrm{f}(\mathrm{h})}\right)$ in den Unterböden $\leq 60 \mathrm{~cm}$ Tiefe von (Pseudogley-)Parabraunerden $(\mathrm{n}=14)$ und Norm-Pseudogleyen $(\mathrm{n}=15)$ aus dem Östlichen Hügelland und der Hohen Geest Schleswig-Holsteins unter Ackernutzung über die Zeit (Datenbank: LLUR S.-H.).

Figure 5. Changes of the horizontal hydraulic conductivity $\left(\mathrm{k}_{\mathrm{f}(\mathrm{h})}\right)$ in arable subsoils $\leq 60 \mathrm{~cm}$ of (Stagnic) Luvisols $(\mathrm{n}=14)$ and Stagnosols $(\mathrm{n}=15)$ in the Weichselian glacial area and Higher Geest region of Schleswig-Holstein over time (data basis: LLUR S.-H).

(Plattengefüge) infolge der fortschreitenden Mechanisierung der Landwirtschaft und zunehmenden Beanspruchung durch die steigenden Maschinengewichte in den Datensätzen nicht abgebildet werden können.

Für die untersuchten Böden der anderen Hauptnaturräume geht aus Tabelle 1 hervor, dass die horizontale Anisotropie in Unterböden des Östlichen Hügellands und der Hohen Geest unter Ackernutzung in $\leq 40 \mathrm{~cm}$ Tiefe am stärksten ausgeprägt ist und für die tonreicheren Böden ( $\geq 12 \%$ Ton) jeweils zunimmt. In $\leq 60 \mathrm{~cm}$ Tiefe ist der Tongehaltseffekt jedoch nur noch für die Unterböden der Hohen Geest auszumachen. Eine Ursache könnte der zunehmende Anteil geogener und pedogener Dichtlagerungen in dieser Tiefe sein, der mögliche anthropogene Effekte überlagert. So stehen bspw. in Böden des Östlichen Hügellands in dieser Tiefe $(\leq 60 \mathrm{~cm})$ häufig von Natur aus dichtgelagerte Tonanreicherungshorizonte (Bt bzw. Sd-Bt der (Pseudogley-) Parabraunerden) an, während derartige natürliche Einflüsse in der Hohen Geest in Form von geogenen Schichtungen (z. B. Geschiebedecksand über Geschiebelehm) erst in tieferen Lagen $(>60 \mathrm{~cm})$ zu erwarten sind. Werden nur die horizontalen $\mathrm{k}_{\mathrm{f}}$-Werte der nicht von Natur aus verdichteten Bodenhorizonte im Östlichen Hügelland (z. B. Al-, Sw-, Mund B-Horizonte) berücksichtigt und über die Zeit betrachtet (Abbildung 3), so wird deutlich, dass die horizontale Anisotropie dieser Böden unabhängig vom Tongehalt tendenziell zugenommen hat. Für verdichtungsempfindliche, tonreichere Böden ( $\geq 12 \%$ Ton) sind die Zunahmen über die Zeit sogar besonders deutlich ausgeprägt.

Auf den Böden der Hohen Geest, die seit Ende der 1970erJahre verstärkt für die Milchvieherzeugung genutzt werden
(Statistikamt Nord, MELUND, 2018), ist auch zu diskutieren, welche Bodenbelastungen durch den intensiven Maschineneinsatz bei der Futterernte oder Wirtschaftsdüngerausbringung sowie durch Trittbelastungen auf Weideflächen zu anthropogenen Strukturänderungen geführt haben. Denn selbst unter Grünlandnutzung weisen diese Geestböden bis in eine Tiefe von $60 \mathrm{~cm}$ eine im Mittel stärker horizontal ausgerichtete Anisotropie auf. Strukturschädigungen unter Grünland ließen sich schon mehrfach nachweisen (Mulholland' und Fullen, 1991; Peth, 2004; Holthusen et al., 2018), wobei die Gefahr gerade auf den für die Grünlandnutzung bevorzugten grund- und stauwasserbeeinflussten und damit sehr verdichtungsempfindlichen Standorten (hydromorphe Böden) als hoch einzustufen ist. Demzufolge ist eine aussagekräftige Bewertung der Anisotropieeffekte in den einzelnen Hauptnaturräumen generell erst unter Berücksichtigung der unterschiedlichen Bodentypen möglich. Nur eine nicht durch geogene Schichtung bzw. pedogene Verdichtung bedingte horizontale Anisotropie in Böden mit einer natürlichen Genese ist somit Ausdruck einer irreversiblen Bodendegradation und führt in der Folge in geneigtem Gelände zu einem entsprechend erhöhten lateralen Massentransport in Senken, Vorfluter, und letztendlich in natürliche Fließgewässer (van der Ploeg et al., 2000; Rogger et al., 2017; Alaoui et al., 2018).

Wenn der Wasserfluss bevorzugt auf dem Plattengefüge der Pflugsohle erfolgt, bedeutet dies gleichzeitig, dass die Nachlieferung von kapillar aufsteigendem Wasser in die Ackerkrume durch das Vorliegen eines solchen wurzelbegrenzenden und in vertikaler Richtung schlecht wasserleitenden Plattengefüges häufig unzureichend ist, was u. a. 
auch zu den im Sommer 2018 sehr deutlichen Trockenschäden beigetragen haben kann.

Für ein Aufbrechen dieser Plattenstrukturen empfiehlt sich gerade unter sehr trockenen Bodenbedingungen eher eine bis in $40 \mathrm{~cm}$ Tiefe lockernde, nicht wendende Bodenbearbeitung (z. B. mittels Paraplow), um die vertikale Wasserleitfähigkeit und die Durchwurzelbarkeit im Pflugsohlenbereich wieder zu erhöhen. Sollte der Einsatz eines entsprechenden Lockerungsgerätes nicht möglich sein, könnten durch die gezielte Heterogenisierung in Form von vertikalen Röhren kontinuierliche und von der Pflanzenwurzel bevorzugt genutzte sowie wie für die Belüftung und Wasserableitung wirksame Poren bis in größere Tiefe geschaffen werden (Jayawardane und Stewart, 1994; Zhai und Horn, 2018a und b). Im Zusammenhang mit wiederholt durchgeführten Meliorations- bzw. Gesundungskalkungen könnte durch die Anwendung von Branntkalk $(\mathrm{CaO})$ zudem eine verstärkte Austrocknung und damit einhergehende Schrumpfrissbildung erreicht werden (Mordhorst et al., 2018). Schließlich ist auch durch den mindestens zweijährigen Anbau von Luzerne mit einem entsprechend tiefreichenden Wasserentzug und dadurch induzierter Schrumpfrissbildung zu rechnen, wodurch eine bessere Durchwurzelung, Wasser- und Gasleitung sowie verbesserte Erreichbarkeit von im Untergrund vorhandenen Nährstoffen erreicht werden kann (Uteau et al., 2013).

\section{Schlussfolgerung}

Bodendeformationen durch mechanische Belastungen können zu einer deutlichen horizontalen Anisotropie des Porensystems im Boden als Folge der anthropogen induzierten Plattenstrukturbildung führen. Inwieweit sich solche negativen Strukturänderungen in den landwirtschaftlich genutzten Böden Schleswig-Holsteins zeigen, wurde anhand eines Datensatzes des LLUR zu vertikalen und horizontalen gesättigten Wasserleitfähigkeiten analysiert.

Die vorhandenen Datensätze machen deutlich, dass die Unterböden unter Ackernutzung im Mittel eine horizontal signifikant ausgeprägte Anisotropie im Bereich der Pflugsohle $(\leq 40 \mathrm{~cm})$ aufweisen, wobei das Ausmaß der Anisotropie in den Hauptnaturräumen und in Abhängigkeit vom Tongehalt variiert. Die tendenziell festgestellte horizontale Anisotropie nicht von Natur aus verdichteter Bodenhorizonte (Al-, Sw-, M-Horizonte) bestärkt den Verdacht, dass anthropogene Strukturänderungen (Plat- tenbildung) durch die im Laufe der Zeit angestiegenen Maschinengewichte ebenso wie häufigere Befahrungen auch bei ungünstigeren Witterungswetterverhältnissen stattgefunden haben. Eine standortangepasste Bodenbewirtschaftung durch entsprechende Landmaschinen ist zwingend erforderlich, um die Bodenfunktionen auch langfristig zu erhalten.

\section{Danksagung}

Die Autoren bedanken sich für die Bereitstellung der Daten aus der Datenbank des Landesamtes für Landwirtschaft, Umwelt und ländliche Räume Schleswig-Holstein (LLUR).

\section{Literatur}

Ad-hoc-AG Boden (2005): Bodenkundliche Kartieranleitung, 5. Aufl., Bundesanstalt für Geowissenschaften und Rohstoffe, Hannover.

Alaoui, A., Rogger, M., Peth, S. und G. Blöschl (2018): Does soil compaction increase floods? A review. Journal of Hydrology 557, 631-642.

Beck-Broichsitter, S., Fleige, H. und R. Horn (2017): Waste capping systems processes and consequences for the longterm impermeability. In: Levin, M., Kim, H.J., Morel, J.L., Burghardt, W., Charzynski, P. und R.K. Shaw (Hrsg.): Soils within Cities. Schweizerbart, Stuttgart, 148-151.

Bertolino, A.V.F.A., Fernandes, N.F., Miranda, J.P.L, Souza, A.P., Lopes, M.R.S. und F. Palmieri (2010): Effects of plough pan development on surface hydrology and on soil physical properties in Southeastern Brazilian plateau. Journal of Hydrology 393, 94-104.

Bogunovic, I., Pereira, P., Kisic, I., Sajko, K. und M. Sraka (2018): Tillage management impacts on soil compaction, erosion and crop yield in Stagnosols (Croatia). Catena 160, 376-384.

Bundesamt für Kartographie und Geodäsie (BKG) (2010): Vektordaten des digitalen Landschaftsmodells, 1: 250.000 .

DeLaune, P.B. und J.W. Sij (2012): Impact of tillage on runoff in long term no-till wheat systems. Soil and Tillage Research 124, 32-35.

Dörner, J. und R. Horn (2009): Direction-dependent behaviour of hydraulic and mechanical properties in struc- 
tured soils under conventional and conservation tillage. Soil and Tillage Research 102, 225-232.

Dörner, J. und R. Horn (2006): Anisotropy of pore functions in structured Stagnic Luvisols in the Weichselian moraine region in N Germany. Journal of Plant Nutrition and Soil Science 169, 213-220.

Duttmann, R., Kuhwald, M. und J. Brunotte (2016): Schutzmaßnahmen gegen Bodenerosion. In: Gute fachliche Praxis - Bodenfruchtbarkeit. AID-Infodienst Ernährung, Landwirtschaft, Verbraucherschutz e. V., Bonn, Deutschland.

Hartge, K. H. und R. Horn (2014): Einführung in die Bodenphysik. 4. Aufl., Schweizerbart, Stuttgart.

Hartge, K.H. (1966): Ein Haubenpermeameter zum schnellen Durchmessen zahlreicher Stechzylinderproben. Zeitschrift für Kulturtechnik und Flurbereinigung 7, 155-163.

Holthusen D., Fleige, H., Brandt, A., Zink A., Horn, R. und J. Reichert (2018): Soil functions and in situ stress distribution in subtropical soils as affected by land use, vehicle type, tire inflation pressure and plant residue removal. Soil and Tillage Research 184, 78-92.

Horn, R. (2015): Soil Compaction and consequences of soil deformation on changes in soil functions. In: S. Nortcliff (Hrsg.): Task Force: Soil matters - Solutions under Foot. GeoEcology Essays, Catena, S. 28-33.

Horn, R. und S. Peth (2011): Mechanics of Unsaturated Soils for Agricultural Applications. In: Huang, P.M., Li, Y. und M.E. Sumner (Hrsg.): Handbook of Soil Sciences. 2. Aufl., CRC, S. 1-30.

Horn, R. und H. Fleige (2003): Risk assessment of subsoil compaction for arable soils in Northwest Germany at farm scale. Soil and Tillage Research 102, 201-208.

Horn, R. (1994): Effect of aggregation of soils on water, gas and heat transport. In: Schulze, E.D. (Hrsg.): Flux control in biological systems. Academic Press, 335-364.

Horn, R. (1981): Die Bedeutung der Aggregierung von Böden für die mechanische Belastbarkeit in dem für Tritt relevanten Auflastbereich und deren Auswirkungen auf physikalische Bodenkenngrößen. Schriftenreihe des FB 14 der TU Berlin.

IPCC (2014): Climate Change 2014: Synthesis Report. Contribution of Working Groups I, II and III to the Fifth Assessment Report of the Intergovernmental Panel on Climate Change [Core Writing Team, R.K. Pachauri and L.A. Meyer (Hrsg.)]. IPCC, Geneva, Switzerland.

Glinski, J., Horabik, J. und J. Lipiec (2011): Encyclopedia of Agrophysics. Springer, Dordrecht, The Netherlands.
Jayawardane, N.S. und B.A. Stewart (Hrsg.) (1994): Subsoil Management Techniques. Advances in Soil Science, CRC, Boca Raton, Florida, USA.

Jones, R.J.A., Spoor, G. und A.J. Thomasson (2003): Vulnerability of subsoils in Europe to compaction: a preliminary analysis. Soil and Tillage Research 73, 131-143.

Kim, H., Anderson, S., Motavalli, P. und C. Gantzer (2010): Compaction effects on soil macropore geometry and related parameters for an arable field. Geoderma 160, 244-251.

Kretzschmar, R. (1996): Kulturtechnisch-bodenkundliches Praktikum. Ausgewählte Labor- und Feldmethoden. Eine Anleitung zum selbständigen Arbeiten an Böden und Gewässern in zwei Bändern, 9. Aufl., Akademisches Institut für Wasserwirtschaft und Landschaftsökologie der Christian-Albrechts-Universität Kiel.

MELUND (2018): Regionale Veränderung des Milchviehbestandes nach Naturräumen. Daten vom Statistikamt Nord, aufbereitet vom Ministerium für Energiewende, Landwirtschaft, Umwelt, Natur und Digitalisierung: Landwirtschafts- und Umweltatlas. http://www.umweltdaten.landsh.de/

Mordhorst, A., Zimmermann, I., Fleige, H. und R. Horn (2018): Improvement of oxygen transport functions in grave soils due to quicklime application depending on soil texture. Geoderma 331, 18-28.

Mordhorst, A., Fleige, H., Zimmermann, I., Burbaum, B., Filipinski, M., Cordsen, E. und R. Horn (2018): Organische Kohlenstoffvorräte von Bodentypen in den Hauptnaturräumen Schleswig-Holsteins (Norddeutschland). Die Bodenkultur: Journal of Land Management, Food and Environment 69, 85-95.

Mulholland, B. und M.A. Fullen (1991): Cattle trampling and soil compaction on loamy sands. Soil Use and Management 7, 189-193.

Pagliai, M., Vignozzi, N. und S. Pellegrini (2004): Soil structure and the effect of management practices. Soil and Tillage Research 79, 131-143.

Peth, S. (2004): Bodenphysikalische Untersuchungen zur Trittbelastung von Böden bei der Rentierweidewirtschaft an borealen Wald- und subarktisch-alpinen Tundrenstandorten - Auswirkungen auf thermische, hydraulische und mechanische Bodeneigenschaften. Schriftenreihe d. Instituts für Pflanzenernährung und Bodenkunde, Nr. 64, Christian-Albrechts-Universität zu Kiel, Deutschland.

Peth, S., Horn, R., Fazekas, O. und B. Richards (2006): Heavy soil loading and its consequences for soil struc- 
ture, strength and deformation of arable soils. Journal of Plant Nutrition and Soil Science 169, 775-783.

R Core Team (2018). R: A Language and Environment for Statistical Computing. R Foundation for Statistical Computing, Vienna, Austria.

Rogger, M., Agnoletti, M. Alaoui, A., Bathurst, J. C., Bodner, G., Borga, M., Chaplot, V., Gallart, F., Glatzel, G., Hall, J., Holden, J., Holko, L., Horn, R., Kiss, A., Kohnová, S., Leitinger, G., Lennartz, B., Parajka, J., Perdigão, R., Peth, S., Plavcová, L., Quinton, J. N., Robinson, M., Salinas, J. L., Santoro, A., Szolgay, J., Tron, S., van den Akker, J. J. H., Viglione, A. und G. Blöschl (2017): Land use change impacts on floods at the catchment scale: Challenges and opportunities for future research. Water Resources Research 53, 5209-5219.

United Nations, Department of Economic and Social Affairs, Population Division (2017). World Population Prospects: The 2017 Revision, Key Findings and Advance Tables. Working Paper No. ESA/P/WP/248.

Uteau, D., Pagenkemper, S.K., Peth, S. und R. Horn (2013): Root and time dependent soil structure formation and its influence on gas transport in the subsoil. Soil and Tillage Research 132, 69-76.

Van der Ploeg, R.R., Machulla, G., Hermsmeyer, D., Ilsemann, J., Gieska, M. und J. Bachmann (2002): Changes in land use and the growing number of flash floods in Germany. IAHS-AISH Publication 273, 317-321.

Van der Ploeg, R.R und P. Schweigert (2001): Elbe river flood peaks and postwar agricultural land use in East Germany, Naturwissenschaften 88, 522-525.
Van der Ploeg, R.R., Hermsmeyer, D. und J. Bachmann (2000): Postwar changes in land use in former West Germany and the increased number of inland floods. Flood Issues in Contemporary Water Management $71,115-123$.

Van der Ploeg, R.R., Ehlers, W. und F. Sieker (1999): Floods and Other Possible Adverse Environmental Effects of Meadowland Area Decline in Former West Germany. Naturwissenschaften, 86, 313-319.

Weißkopf, P., Zielmann, U., Wiermann, C., Horn, R., Anken, Th. und E. Diserens (2000): Influences of conventional and onland-ploughing on soil structure. Advances in Geoecology 32, 73-82.

Zhai, X. und R. Horn (2018a): Effect of static and cyclic loading including spatial variation caused by vertical holes on changes in soil aeration. Soil and Tillage Research, 177, 61-68.

Zhai, X. und R. Horn (2018b): Dynamics of pore functions and gas transport parameters in artificially ameliorated soils due to static and cyclic loading. Geoderma, im Druck (in press).

Zink, A. (2009): Bodenstabilität und Auswirkungen dynamischer Lasteinträge auf physikalische Eigenschaften von Ackerböden unter konservierender und konventioneller Bodenbearbeitung. Schriftenreihe des Instituts für Pflanzenernährung und Bodenkunde, Nr. 84, Christian-Albrechts-Universität zu Kiel, Deutschland. 